\title{
ZERO-HOPF PERIODIC ORBITS FOR A RÖSSLER DIFFERENTIAL SYSTEM
}

\author{
JAUME LLIBRE $^{1}$ AND AMMAR MAKHLOUF ${ }^{2}$
}

\begin{abstract}
We study the zero-Hopf bifurcation of the Rössler differential system

$$
\dot{x}=x-x y-z, \quad \dot{y}=x^{2}-a y, \quad \dot{z}=b(c x-z) .
$$

where the dot denotes derivative with respect to the independent variable $t$ and $\mathrm{a}, \mathrm{b}, \mathrm{c}$ are real parameters.
\end{abstract}

\section{Introduction AND STATEMENT OF the MAin RESUlt}

Rössler, using the geometry of the 3-dimensional flows, introduced several differential systems as prototypes of the simplest autonomous differential equations exhibiting chaos. The simplicity of his systems is in the sense of minimal dimension, minimal number of parameters and minimal nonlinearities. Nowadays in MathSciNet appear more than 114 articles with the words Rössler system in the title. In 2006 Letellier, Roulin and Rössler [2] did a classification of chaotic attractors in $\mathbb{R}^{3}$ in their classification they go back to the differential system

$$
\begin{aligned}
& \dot{x}=x-x y-z, \\
& \dot{y}=x^{2}-a y, \\
& \dot{z}=b(c x-z),
\end{aligned}
$$

already considered by Rössler [2, 5, 6] in 1977, showing that this system exhibits a pure cut chaos in their terminology. As usual the dot denotes derivative with the time t. This differential system has three families of zero-Hopf equilibria. Our objective is to study if from these equilibria it bifurcate some periodic orbits.

Our interest in system (1) is motivated due to the fact that frequently the complex dynamics of some chaotic nonlinear systems started in their equilibria. More precisely, Cândido and Llibre in [1] studied the existence of zero-Hopf bifurcations in 3-dimensional systems, and numerically they show that such bifurcations sometimes are the starting bifurcation of a route to the chaotic motion.

Note that system (1) is invariant under the symmetry $(x, y, z) \rightarrow(-x, y,-z)$. A zero-Hopf equilibrium is an equilibrium point of a 3-dimensional autonomous differential system, which has a zero eigenvalue and a pair of purely imaginary eigenvalue.

Proposition 1. There are two one-parameter families of systems (1) for which this system has a zero-Hopf equilibrium point namely the origin when $a=0, b=1, c=$

2010 Mathematics Subject Classification. 37G15, 37C80, 37C30.

Key words and phrases. periodic orbit,Rössler differential system, averaging theory. 
$1+\beta^{2}$ with $\beta>0$, and the two equilibria $(x, y, z)=( \pm \sqrt{a(1-c)}, 1-c, \pm c \sqrt{a(1-c)})$ when $c=a, b=0$, and $(2-3 a) a>0$.

A zero-Hopf bifurcation is a two-parameter unfolding (or family) of a 3-dimensional autonomous differential system with a zero-Hopf equilibrium. Here we are mainly interested in the periodic solutions which can bifurcate from a zero-Hopf equilibrium point of system (1). Our main results on the periodic solutions of the differential system (1) are the following.

Theorem 2. Let $(a, b, c)=\left(\varepsilon a_{1}, 1+\varepsilon b_{1}, 1+\beta^{2}+\varepsilon c_{1}\right)$ where $\varepsilon$ is a small parameter. Then the Rössler system (1) has a zero-Hopf bifurcation at the zero-Hopf equilibrium point localized at the origin of coordinates, and a periodic orbit borns at this equilibrium when $\varepsilon=0$ and it exists for $\varepsilon>0$ sufficiently small.

Theorem 3. Let $(b, c)=\left(\varepsilon b_{1}, a+\varepsilon c_{1}\right)$ where $\varepsilon$ is a sufficiently small parameter. By using the averaging theory we cannot detect periodic orbits bifurcating from the zeroHopf equilibria localized at the points $(x, y, z)=( \pm \sqrt{a(1-c)}, 1-c, \pm c \sqrt{-a(c-1)})$.

Theorems 2 and 3 are proved in section 2 and in section 3 , respectively. Their proofs are based in the averaging theory for computing periodic orbits, see the appendix. For others applications of the averaging theory for studying limit cycles, see for instance $[1,3,4]$.

\section{Proof of proposition 1 And Theorem 2}

Proof of Proposition 1. System (1) possesses the equilibrium points (0,0,0) and $( \pm \sqrt{a(1-c)}, 1-c, \pm c \sqrt{a(1-c)})$, i.e. system (1) has three equilibria if $a(1-c)>0$ two equilibria if $a=0$ and $c \neq 1$, and one equilibria otherwise. The Jacobian matrix of system (1) is

$$
\left(\begin{array}{ccc}
1-y & -x & -1 \\
2 x & -a & 0 \\
b c & 0 & -b
\end{array}\right)
$$

The characteristic polynomial which correspond to the equilibrium point $(0,0,0)$ is $p(\lambda)=-\lambda^{3}+(1-a-b) \lambda^{2}+(a+b-a b-b c) \lambda-a b(c-1)$. In order to study the zero-Hopf bifurcation we impose that $p(\lambda)=-\lambda\left(\lambda^{2}+\beta^{2}\right)$ with $\beta>0$, and we obtain one family of zero-Hopf equilibrium points when $a=0, b=1, c=1+\beta^{2}$.

The characteristic polynomial which correspond to the equilibria $( \pm \sqrt{a(1-c)}, 1-$ $c, \pm c \sqrt{a(1-c)})$ is $q(\lambda)=-\lambda^{3}+(-a-b+c) \lambda^{2}-a(2+b-3 c) \lambda+2 a b(c-1)$. In order to study the zero-Hopf bifurcation we impose that $q(\lambda)=-\lambda\left(\lambda^{2}+\beta^{2}\right)$ with $\beta>0$, we obtain one family of zero-Hopf equilibria when $c=a, b=0$ and $\beta=\sqrt{(2-3 a) a}$ if $(2-3 a) a>0$.

We shall see that from the zero-Hopf equilibrium $(0,0,0)$ one periodic orbit bifurcate in a zero-Hopf bifurcation. 
Proof of Theorem 2. If $(a, b, c)=\left(\varepsilon a_{1}, 1+\varepsilon b_{1}, 1+\beta^{2}+\varepsilon c_{1}\right)$ with $\varepsilon \neq 0$ a sufficiently small parameter, then system (1) becomes

$$
\begin{aligned}
& \dot{x}=x-x y-z, \\
& \dot{y}=x^{2}-\varepsilon a_{1} y, \\
& \dot{z}=\left(1+\varepsilon b_{1}\right)\left(\left(1+\beta^{2}+\varepsilon c_{1}\right) x-z\right) .
\end{aligned}
$$

We need to write the linear part of system (2) at the equilibrium point $(0,0,0)$ in its real Jordan normal form, i.e. into the form

$$
\left(\begin{array}{ccc}
0 & -\beta & 0 \\
\beta & 0 & 0 \\
0 & 0 & 0
\end{array}\right) \text {. }
$$

In the new variables $(X, Y, Z)$ defined by $(x, y, z)=(X, Z, X+\beta Y)$ we obtain the differential system

$$
\begin{aligned}
& \dot{X}=-\beta Y-X Z \\
& \dot{Y}=\beta X+\frac{1}{\beta} X Z+\varepsilon\left(\left(\beta b_{1}+\frac{c_{1}}{\beta}\right) X-b_{1} Y\right)+\varepsilon^{2} \frac{1}{\beta} b_{1} c_{1} X, \\
& \dot{Z}=-\varepsilon a_{1} Z+X^{2} .
\end{aligned}
$$

Consider the change to cylindrical coordinates $(r, \theta, Z)$ defined by $X=r \cos \theta, Y=$ $r \sin \theta$ we obtain the differential system

(4)

$$
\begin{aligned}
& \dot{r}=\frac{1}{\beta}(r Z \cos \theta(\sin \theta-\beta \cos \theta))+\varepsilon \frac{1}{\beta} r \sin \theta\left(\left(c_{1}+b_{1} \beta^{2}\right) \cos \theta-b_{1} \beta \sin \theta\right), \\
& \dot{\theta}=\frac{1}{2 \beta}\left(2 \beta^{2}+(1+\cos 2 \theta+\beta \sin 2 \theta) Z\right)+\varepsilon \cos \theta\left(\left(\frac{c_{1}}{\beta}+b_{1} \beta\right) \cos \theta-b_{1} \sin \theta\right), \\
& \dot{Z}=r^{2} \cos ^{2} \theta-\varepsilon a_{1} Z .
\end{aligned}
$$

We take $\theta$ as the new independent variable of the differential system and we get (5)

$$
\begin{aligned}
& r^{\prime}=\frac{2}{A}(2 r Z \cos \theta(\sin \theta-\beta \cos \theta))+\varepsilon \frac{4}{A^{2}}\left(r \beta(Z \cos \theta+\beta \sin \theta)\left(c_{1}+b_{1} \beta^{2}\right) \cos \theta-b_{1} \beta \sin \theta\right), \\
& Z^{\prime}=\frac{1}{A} 2 \beta r^{2} \cos ^{2} \theta-\varepsilon \frac{2}{A^{2}} \beta\left(2 r^{2}\left(c_{1}+b_{1} \beta^{2}\right) \cos ^{4} \theta-2 \beta b_{1} \cos ^{3} \theta \sin \theta r^{2}+a_{1} Z A\right),
\end{aligned}
$$

where $A=2 \beta^{2}+(1+\cos 2 \theta+\beta \sin 2 \theta) Z$.

Here the prime denotes the derivative with respect to the variable $\theta$. Since we want to study the zero-Hopf bifurcation we scale the variables $(r, Z)$ with the small parameter $\varepsilon$ and we shall study the periodic solutions of the scaled differential system. These periodic solutions, if they exist, will tend to the origin when $\varepsilon$ tends to zero and consequently there will be periodic solutions in a zero-Hopf bifurcation at the origin. Hence by putting $(r, Z)=(\varepsilon R, \varepsilon U)$, we obtain the following system. (6)

$$
\begin{aligned}
& R^{\prime}=\varepsilon \frac{R}{\beta^{2}}\left(\left(c_{1}+b_{1} \beta^{2}+U\right) \cos \theta \sin \theta-\beta b_{1} \sin ^{2} \theta-\beta U \cos ^{2} \theta\right)=\varepsilon F_{1}(\theta, R, U), \\
& U^{\prime}=\varepsilon \frac{1}{\beta\left(R^{2} \cos ^{2} \theta-a_{1} U\right)}=\varepsilon F_{2}(\theta, R, U) .
\end{aligned}
$$

We shall apply the averaging theory described in the appendix to the differential system (6). Using the notation of the appendix, we have $t=\theta, T=2 \pi, x=(R, U)^{T}$ 
and

where

$$
F(\theta, R, U)=\left(\begin{array}{c}
F_{1}(\theta, R, U) \\
F_{2}(\theta, R, U)
\end{array}\right), \quad f(R, U)=\left(\begin{array}{c}
f_{1}(R, U) \\
f_{2}(R, U)
\end{array}\right)
$$

$$
\begin{aligned}
& f_{1}(R, U)=\frac{1}{2 \pi} \int_{0}^{2 \pi} F_{1}(\theta, R, U) d \theta, \\
& f_{2}(R, U)=\frac{1}{2 \pi} \int_{0}^{2 \pi} F_{2}(\theta, R, U) d \theta .
\end{aligned}
$$

It is immediate to check that differential system (6) satisfies all the assumptions of Theorem 4 of the appendix. So we will apply it to system (6). Now we compute the integrals (15), i.e. We obtain

$$
f_{1}(R, U)=-\frac{R\left(b_{1}+U\right)}{2 \pi \beta}, \quad f_{2}(R, U)=-\frac{R^{2}-2 a_{1} U}{4 \pi \beta} .
$$

The system $f_{1}(R, U)=0, f_{2}(R, U)=0$ has a unique solution $\left(R^{*}, U^{*}\right)$ with $R^{*}>0$, namely $\left(R^{*}, U^{*}\right)=\left(\sqrt{-2 a_{1} b_{1}},-b_{1}\right)$ if $a_{1} b_{1}<0$. The corresponding Jacobian (16) at $\left(R^{*}, U^{*}\right)$ takes the value $\frac{-a_{1} b_{1}}{\beta^{2}}>0$. Theorem 4 guarantees for $\varepsilon$ sufficiently small the existence of a periodic solution $(R(\theta, \varepsilon), U(\theta, \varepsilon))$ of system $(6)$ such that $(R(0, \varepsilon), U(0, \varepsilon)) \longrightarrow\left(R^{*}, U^{*}\right)$ when $\varepsilon \longrightarrow 0$. That is system (6) has the periodic solution

$$
(R(\theta, \varepsilon), U(\theta, \varepsilon))=\left(R^{*}+O(\varepsilon), U^{*}+O(\varepsilon)\right) .
$$

So system (5) has a periodic solution

$$
(r(\theta, \varepsilon), Z(\theta, \varepsilon))=\left(\varepsilon R^{*}+O\left(\varepsilon^{2}\right), \varepsilon U^{*}+O\left(\varepsilon^{2}\right)\right),
$$

and consequently system (4) has a periodic solution

$$
(r(t, \varepsilon), \theta(t, \varepsilon), Z(t, \varepsilon))=\left(\varepsilon R^{*}+O\left(\varepsilon^{2}\right), \beta t+O(\varepsilon), \varepsilon U^{*}+O\left(\varepsilon^{2}\right)\right) .
$$

Therefore system (3) has a periodic solution

$$
(X(t, \varepsilon), Y(t, \varepsilon), Z(t, \varepsilon))=\left(\varepsilon R^{*} \cos (\beta t)+O\left(\varepsilon^{2}\right), \varepsilon R^{*} \sin (\beta t)+O\left(\varepsilon^{2}\right), O\left(\varepsilon^{2}\right)\right) .
$$

Finally system $(2)$ has a periodic solution

$$
(x(t, \varepsilon), y(t, \varepsilon), z(t, \varepsilon))=\left(\varepsilon R^{*} \cos (\beta t)+O\left(\varepsilon^{2}\right), O\left(\varepsilon^{2}\right), \varepsilon R^{*}\left(\cos (\beta t)+\beta \sin (\beta t)+O\left(\varepsilon^{2}\right)\right)\right. \text {. }
$$

This completes the proof of the theorem.

\section{Proof of Theorem 3}

Proof of Theorem 3. Consider the second equilibrium point $(x, y, z)=(-\sqrt{a(1-c)}, 1-$ $c,-c \sqrt{a(1-c)})$ with $a(1-c)>0$. We assume that $a(1-c)>0$ and $(2-3 a) a>0$. The corresponding second family of perturbed parameters is $(b, c)=\left(\varepsilon b_{1}, a+\varepsilon c_{1}\right)$. We translate this equilibrium point to the origin $(x, y, z) \mapsto(x-\sqrt{a(1-c)}, y+1-$ $c, z-c \sqrt{a(1-c)}$ ), and we obtain the differential system

$$
\begin{aligned}
& \dot{x}=-z+\left(a-y+c_{1} \varepsilon\right) x+\sqrt{a\left(1-a-\varepsilon c_{1}\right)} y, \\
& \dot{y}=-2 \sqrt{a\left(1-a-\varepsilon c_{1}\right)} x-a y+x^{2}, \\
& \dot{z}=\varepsilon b_{1}\left(a+\varepsilon c_{1}\right) x+\varepsilon b_{1} z .
\end{aligned}
$$


The Jacobian matrix at the origin and for $\varepsilon=0$ is

$$
\left(\begin{array}{ccc}
a & \sqrt{(1-a) a} & -1 \\
-2 \sqrt{(1-a) a} & -a & 0 \\
0 & 0 & 0
\end{array}\right)
$$

Its eigenvalues are $(0, \pm \sqrt{(2-3 a) a} i)$. We need to write the linear part of system $(2)$ at the equilibrium point $(0,0,0)$ in its real Jordan normal form, i.e. into the form

$$
\left(\begin{array}{ccc}
0 & -\sqrt{(2-3 a) a} & 0 \\
\sqrt{(2-3 a) a} & 0 & 0 \\
0 & 0 & 0
\end{array}\right) .
$$

In the new variables $(X, Y, Z)$ defined by

(8)

$$
\begin{aligned}
& x=X+\frac{Z}{3 a-2}, \\
& y=\frac{3 a^{2} \sqrt{2-3 a} X-4 \sqrt{a} Y+12 a^{3 / 2} Y-9 a^{5 / 2} Y+2 \sqrt{2-3 a}(a X+(1+a) Z)}{(2-3 a)^{3 / 2} \sqrt{(1-a) a}}, \\
& z=Z
\end{aligned}
$$

we obtain the differential system

(9)

$$
\begin{aligned}
\dot{X}= & \frac{a X^{2}}{\sqrt{(1-a) a}}+\frac{2 \sqrt{1-a} Z^{2}}{(2-3 a)^{2} \sqrt{a}}+Y\left(-\frac{Z}{\sqrt{(2-3 a)(1-a)}}-\frac{\sqrt{(2-3 a) a\left(1-a-c_{1} \varepsilon\right)}}{\sqrt{1-a}}\right) \\
& +X\left(a+\frac{\sqrt{2-3 a} Y}{\sqrt{1-a}}+\frac{(2-a) Z}{\sqrt{(1-a) a}(3 a-2)}+\varepsilon c_{1}-\frac{a \sqrt{a\left(1-a-c_{1} \varepsilon\right)}}{\sqrt{(1-a) a}}-\frac{b_{1} \varepsilon\left(a+\varepsilon c_{1}\right)}{3 a-2}\right) \\
& +\frac{Z}{(2-3 a)^{2} a}\left(4 \sqrt{(1-a) a^{2}\left(1-a-\varepsilon c_{1}\right)}+a\left(-6 a^{2}-\left(2+\varepsilon b_{1}\right)\left(2+\varepsilon c_{1}\right)\right.\right. \\
& \left.\left.-6 \sqrt{(1-a) a^{2}\left(1-a-\varepsilon c_{1}\right)}+a\left(10+2 \varepsilon b_{1}+3 \varepsilon c_{1}\right)\right)\right), \\
\dot{Y}= & -\frac{X^{2}}{\sqrt{(2-3 a)(1-a)}}-\frac{3 \sqrt{1-a} Z^{2}}{(2-3 a)^{5 / 2}}+Y\left(-a+\frac{\sqrt{a} Z}{\sqrt{(2-3 a)(1-a)}}+\frac{\sqrt{a^{2}\left(1-a-\varepsilon c_{1}\right)}}{\sqrt{1-a}}\right) \\
& +\frac{1}{(2-3 a)^{3 / 2} \sqrt{(1-a) a}} Z\left(-4 a^{2} \sqrt{1-a}-\varepsilon b_{1} \sqrt{1-a}\left(2+\varepsilon c_{1}\right)-4 \sqrt{a^{2}\left(1-a-\varepsilon c_{1}\right)}\right. \\
& \left.+4 \sqrt{a^{4}\left(1-a-\varepsilon c_{1}\right)}+a \sqrt{1-a}\left(4+2 \varepsilon b_{1}+\varepsilon c_{1}\right)\right)+X\left(-\frac{\sqrt{a} Y}{\sqrt{1-a}}+\frac{(4-3 a) Z}{(2-3 a)^{3 / 2} \sqrt{1-a}}\right. \\
& +\frac{1}{\sqrt{(2-3 a)(1-a) a}}\left(-2 a^{2} \sqrt{1-a}+\varepsilon a \sqrt{1-a}\left(b_{1}-c_{1}\right)+\varepsilon^{2} b_{1} c_{1} \sqrt{1-a}+2 \sqrt{a^{2}\left(1-a-\varepsilon c_{1}\right)}\right. \\
\dot{Z}= & -a^{\left.\left.3 / 2 \sqrt{a\left(1-a-\varepsilon c_{1}\right)}\right)\right)}, \\
& \frac{b_{1} Z\left(2-2 a+\varepsilon c_{1}\right)}{3 a-2}+\varepsilon b_{1}\left(a+c_{1} \varepsilon\right) X .
\end{aligned}
$$

Consider the cylindrical coordinates $(r, \theta, Z)$ defined by $X=r \cos \theta, Y=r \sin \theta$ after we take $\theta$ as the new independent variable and doing the scaling $(r, Z)=$ 
$(\varepsilon R, \varepsilon U)$ we obtain the following system.

$$
\begin{aligned}
& R^{\prime}=\varepsilon \frac{\sqrt{1-a}}{2(2-3 a)^{3} \sqrt{a(1-a)}}\left(\frac{1}{A}+\frac{1}{B}\right)=\varepsilon F_{1}(\theta, R, U), \\
& U^{\prime}=-\varepsilon b_{1} \frac{\sqrt{1-a}(2(1-a) U+a(3 a-2) R \cos \theta)}{(2-3 a)^{3 / 2} \sqrt{a(1-a)}}=\varepsilon F_{2}(\theta, R, U),
\end{aligned}
$$

where

(11)

$$
\begin{aligned}
A= & \sqrt{a(1-a)}(U+(3 a-2) R \cos \theta)\left(-2(2-3 a)^{3 / 2} a R \cos (2 \theta)+6(a-1) \sqrt{a} U \sin \theta\right. \\
& \left.-2 \cos \theta\left(2 \sqrt{2-3 a}(a-1) U+\sqrt{a}\left(9 a^{2}-9 a+2\right) R \sin \theta\right)\right) \\
B= & (a-1) \sqrt{a}\left(4 \sqrt{2-3 a}(a-1) \sqrt{a}(a-1) b_{1}+(3 a-2) c_{1}\right) U \cos \theta \\
& +(2-3 a)^{3 / 2} \sqrt{a}\left(-4 c_{1}+a\left(2(a-1) b_{1}+(8-3 a) c_{1}\right)\right) R \cos ^{2} \theta+(3 a-2) \sin \theta \\
& \left(2(1-a)\left(2(a-1) b_{1}+3 a c_{1}\right) U+a(3 a-2) R\left(2\left((a-1) b_{1}+c_{1}\right) \cos \theta\right.\right. \\
& \left.\left.\left.+\sqrt{a(2-3 a)} c_{1} \sin \theta\right)\right)\right) .
\end{aligned}
$$

As usual the prime denotes derivative with respect to $\theta$. We shall apply the averaging theory described in the appendix to the differential system (10). Using the notation of the appendix, it is immediate to check that differential system (10) satisfies all the assumptions of Theorem 4 of the appendix. So we will apply it to system (10). Now we compute the integrals (15), and we obtain

$$
\begin{aligned}
& f_{1}(R, U)=\frac{R\left(a^{2}\left(b_{1}-3 c_{1}\right)+2 a c_{1}-2 \sqrt{a(1-a)} U\right)}{2 a^{3 / 2}(2-3 a)^{3 / 2}}, \\
& f_{2}(R, U)=\frac{2(a-1) b_{1} U}{\sqrt{a}(2-a)^{3 / 2}} .
\end{aligned}
$$

The system $f_{1}(R, U)=0, f_{2}(R, U)=0$ has the unique solution $\left(R^{*}, U^{*}\right)=(0,0)$. In order to go to averaging of second order, we need that $a=1$, but then $(2-3 a) a<0$, and this is not possible. So with the averaging theory we cannot detect periodic orbits bifurcating from the equilibrium point $(-\sqrt{a(1-c)}, 1-c,-c \sqrt{a(1-c)})$. This completes the proof of the theorem.

\section{APPENDIX:AVERAGING THEORY OF FIRST ORDER}

We work with the two initial value problems

$$
\dot{\mathbf{x}}=\varepsilon F_{1}(t, \mathbf{x})+\varepsilon^{2} F_{2}(t, \mathbf{x}, \varepsilon), \quad \mathbf{x}(0)=\mathbf{x}_{0},
$$

and

$$
\dot{\mathbf{y}}=\varepsilon f(\mathbf{y}), \quad \mathbf{y}(0)=\mathbf{x}_{0},
$$

with $\mathbf{x}, \mathbf{y}$ and $\mathbf{x}_{0}$ in some open $\Omega$ of $\mathbb{R}^{n}, t \in[0, \infty), \varepsilon \in\left(0, \varepsilon_{0}\right]$. Let $\mathbf{F}_{\mathbf{1}}$ and $\mathbf{F}_{\mathbf{2}}$ be periodic functions of period $\mathrm{T}$ in the variable $\mathrm{t}$, and we define

$$
f(\mathbf{y})=\frac{1}{T} \int_{0}^{T} F_{1}(t, \mathbf{y}) d t .
$$

Theorem 4. By hypotheses the functions $F_{1}, D_{\mathbf{x}} F_{1}, D_{\mathbf{x x}} F_{1}$ and $D_{\mathbf{x}} F_{2}$ are continuous, bounded by a constant independent of $\varepsilon$ in $[0, \infty) \times \Omega \times\left(0, \varepsilon_{0}\right]$, and that $y(t) \in \Omega$ for $t \in[0,1 / \varepsilon]$. Then the next statements are satisfied. 
(1) For $t \in[0,1 / \varepsilon]$ it follows that $\mathbf{x}(t)-\mathbf{y}(t)=O(\varepsilon)$ as $\varepsilon \rightarrow 0$.

(2) If $p \neq 0$ is an equilibrium point of system (14) and

$$
\operatorname{det} D_{\mathbf{y}} f(p) \neq 0 \text {, }
$$

then there is a periodic solution $\phi(t, \varepsilon)$ of period $T$ for system (13) such that $\phi(0, \varepsilon)-p=O(\varepsilon)$ as $\varepsilon \rightarrow 0$.

The notation $D_{\mathbf{x}} g$ means the Jacobian matrix of $g$ with respect to the components of $\mathbf{x}$, and $D_{\mathbf{x x}} g$ means the Hessian matrix of $g$.

For a proof of Theorem 4 see [7].

\section{ACKnowledGements}

This work is supported by the Ministerio de Economía, Industria y Competitividad, Agencia Estatal de Investigación grant MTM2016-77278-P (FEDER), the Agència de Gestió d'Ajuts Universitaris i de Recerca grant 2017SGR1617, and the H2020 European Research Council grant MSCA-RISE-2017-777911.

\section{REFERENCES}

[1] M.R. CÂNDIDO AND J. LliBRE, Zero-Hopf bifurcations in 3-dimensional differential systems with no equilibria, Math..Comp. Simulation 151 (2018), 54-76.

[2] C. Letellier, E. Roulin AND O.E. Rössler, Inequivalent topologies of chaos in simple equations, Chaos, Solitons and Fractals 28 (2006), 337-360.

[3] J.LlibRe, Periodic orbits in the zero-Hopf bifurcation of the Rössler system, Romanian Astron. J 24 (2014), 49-60.

[4] J. LlibRe AND X. ZHANG, Hopf bifurcation in higher dimensional differential systems via the averaging method differential system in $\mathbb{R}^{d}$, Pacific J. Math. 240 (2009), 321-341.

[5] E. Rössler, An equation for continuous chaos, Phys. Lett. A, 57 (1976), 397-398.

[6] O.E. Rössler, Continuous chaos-four prototype equations, Ann. New York, Acad.Sci. 316 (1979), 376-392

[7] F. Verhulst, Nonlinear Differential Equations and Dynamical Systems, Universitext, Springer, New York, 1996.

1 Departament de Matematiques, Universitat Autònoma de Barcelona, 08193 Bellaterra, Barcelona, Catalonia, Spain

E-mail address: jllibre@mat.uab.cat

2 Department of Mathematics, University of Annaba, Laboratory LMa, P.O.Box 12 , Annaba 23000, Algeria

E-mail address: makhloufamar@yahoo.fr 